Mappemonde

Revue trimestrielle sur l'image géographique et les formes du territoire

$130 \mid 2021$

Varia

\title{
Sortir hors de la classe : apports didactiques d'un dispositif d'expérimentations sensibles en géographie
}

Out of the classroom: the didactic benefits of a system experiential experiments in geography

Las salidas fuera de clase: aprotaciones didácticas de un instrumento de experimentación sensible en geografía

\section{Sophie Gaujal}

\section{OpenEdition}

Journals

Édition électronique

URL : https://journals.openedition.org/mappemonde/5145

DOI : $10.4000 /$ mappemonde. 5145

ISSN : 1769-7298

\section{Éditeur}

UMR ESPACE

Référence électronique

Sophie Gaujal, «Sortir hors de la classe : apports didactiques d'un dispositif d'expérimentations sensibles en géographie », Mappemonde [En ligne], 130 | 2021, mis en ligne le 15 mars 2021, consulté le 20 mai 2021. URL : http://journals.openedition.org/mappemonde/5145 ; DOI : https://doi.org/ 10.4000/mappemonde.5145

Ce document a été généré automatiquement le 20 mai 2021. 


\title{
Sortir hors de la classe : apports didactiques d'un dispositif d'expérimentations sensibles en géographie
}

\author{
Out of the classroom: the didactic benefits of a system experiential experiments \\ in geography \\ Las salidas fuera de clase: aprotaciones didácticas de un instrumento de \\ experimentación sensible en geografía
}

Sophie Gaujal

Différents travaux se sont penchés, ces quinze dernières années, sur les manières de faire des artistes contemporains avec l'espace et, de manière symétrique, sur les manières de produire du savoir via l'esthétique des géographes de l'art. Anne Volvey en a proposé une synthèse dans son habilitation à diriger des recherches (2012). Mes recherches interrogent un troisième pôle autour duquel ces collaborations peuvent prendre forme, le pôle scolaire. Enseignante dans le secondaire et chercheure en didactique de la géographie, j'en interroge la possibilité et la pertinence par l'expérimentation de différents dispositifs ${ }^{1}$ inspirés de démarches artistiques : sorties sensibles, géo-photographie, cartographie sensible, performance (Gaujal, 2016). Cette posture de praticienne-chercheure n'est pas sans conséquence sur la méthodologie de recherche. Celle-ci se caractérise, en effet, par des va-et-vient incessants entre la recherche et les expérimentations menées en classe, de manière itérative. Les questionnements sont à la fois d'ordre théorique et pragmatique, par exemple: "quelles transformations connaissent les démarches artistiques lorsqu'elles sont introduites dans le cours de géographie?» «de manière symétrique, quels apprentissages permettent-elles aux élèves et comment les évaluer?»; «comment transforment-elles les pratiques enseignantes, et comment les positionner par rapport aux pratiques ordinaires? ». Enfin, elle engage la subjectivité du chercheur, nécessitant 
une vigilance particulière dans l'analyse des résultats, et leur donnant une tonalité spécifique, s'apparentant par moment à un récit réflexif.

2 Cet article se penche plus particulièrement sur l'un des dispositifs expérimentés jusqu'ici, la sortie sensible. Interrogée depuis le champ scientifique par des géographes comme Élise Olmedo (Olmedo, 2012b, 2012a ; Feildel et al. 2016 ; Olmedo, 2017), travaillée dans le champ de la médiation des savoirs par des institutions culturelles comme les CAUE (Conseils d'Architecture, d'Urbanisme et d'Environnement), la sortie sensible a été importée dans le champ scolaire par des travaux en didactique, dans le primaire (Briand, 2014) et le secondaire (Gaujal, 2016, 2017a, 2017b). Cet article, de synthèse, s'appuie sur ces travaux et sur le corpus constitué depuis 2011, composé d'observations de sorties sur le terrain (sensibles ou non, corpus 1 et 2) menées dans le champ scientifique et scolaire, et d'expérimentations (corpus 3), menées essentiellement auprès d'élèves de Première ES du lycée dans lequel j'ai enseigné, dans la banlieue parisienne (tableau 1). Il a pour objectif d'interroger la manière dont la sortie sensible permet d'apprendre l'espace et ainsi de remplir plus efficacement les objectifs associés aux sorties scolaires en géographie. Pour cela, je la rapproche tout d'abord des sorties scolaires ordinaires, dont je propose une définition. J'en explore ensuite, à travers la présentation d'expérimentations menées en classe, la diversité des modalités possibles pour tenter, enfin, d'en dégager quelques principes généraux.

Tableau 1. La sortie sensible scolaire : corpus

\begin{tabular}{|c|c|c|}
\hline & Méthode & Données recueillies \\
\hline \multirow{2}{*}{$\begin{array}{l}\text { Corpus } 1 \\
\text { Observations de } \\
\text { sorties de terrain }\end{array}$} & $\begin{array}{l}\text { Conception et organisation d'un concours de } \\
\text { photographies à l'échelle académique, classes } \\
\text { du secondaire (GéoPhotoGraphes), 2012-2018. }\end{array}$ & $\begin{array}{l}\text { Productions des classes } \\
\text { (180 classes participantes } \\
\text { entre } 2012 \text { et 2018). } \\
\text { Témoignages des } \\
\text { enseignants recueillis par } \\
\text { questionnaire en } 2015 .\end{array}$ \\
\hline & $\begin{array}{l}\text { Concours de cartographie sensible à l'échelle } \\
\text { nationale, classes du secondaire et du } \\
\text { primaire (Cartographie ton Quartier), } \\
\text { animation d'un carnet de bord des classes } \\
\text { publié sur le blog GéoPhotoGraphes\&Carto, } \\
\text { 2014-2016. }\end{array}$ & $\begin{array}{l}\text { Productions des classes } \\
\text { (300 classes participantes } \\
\text { entre 2014 et 2016). } \\
\text { Carnets de bords des } \\
\text { classes (composés des } \\
\text { préparations } \\
\text { enseignants et des } \\
\text { activités des élèves). }\end{array}$ \\
\hline \multirow[b]{2}{*}{$\begin{array}{l}\text { Corpus } 2 \text { Observation } \\
\text { de sorties sensibles }\end{array}$} & $\begin{array}{l}\text { a. En } 2012 \text {, sortie sensible conçue par le CAUE } \\
\text { du } 92 \text { sur l'île Seguin pour une classe de } \\
\text { Première ES. }\end{array}$ & $\begin{array}{l}\text { Observation participante } \\
\text { Recueil des } \\
\text { représentations des } \\
\text { élèves au début et à la fin } \\
\text { de la sortie (" } 5 \text { mots pour } \\
\text { caractériser l'île Seguin ») }\end{array}$ \\
\hline & $\begin{array}{l}\text { b. En mai } 2013 \text {, promenade sensible dans le } \\
\text { cadre du séminaire sur le sensible organisé } \\
\text { par Élise Olmedo }\end{array}$ & Observation participante \\
\hline
\end{tabular}




\begin{tabular}{|c|c|c|}
\hline & $\begin{array}{l}\text { c. En 2014, balade sonore dans le parc de l'île } \\
\text { Monsieur (Sèvres) conçue par Sébastien } \\
\text { Branche, professeur d'arts du son et } \\
\text { musicien-performeur avec une classe de } \\
\text { Terminale. }\end{array}$ & Observation participante \\
\hline \multirow{4}{*}{$\begin{array}{l}\text { Corpus } 3 \text { Conception } \\
\text { de dispositifs de } \\
\text { sorties sensibles avec } \\
\text { des classes de } \\
\text { Première ES à } \\
\text { Boulogne-Billancourt }\end{array}$} & $\begin{array}{l}\text { 2011, sortie-relevé dans les anciens terrains } \\
\text { Renault (classe de Première ES). }\end{array}$ & $\begin{array}{l}\text { Productions des élèves } \\
\text { (60 photographies + notes } \\
\text { d'intention) } \\
\text { Parcours iconographique } \\
\text { de } 10 \text { élèves } \\
\text { Parcours commentés d'un } \\
\text { élève }\end{array}$ \\
\hline & $\begin{array}{l}\text { 2014, sortie-jeu dans la cité du Pont de Sèvres } \\
\text { (classe de Première ES). }\end{array}$ & $\begin{array}{l}\text { Observation participante } \\
\text { Données prélevées par les } \\
\text { élèves } \\
\text { Recueil des } \\
\text { représentations à la fin } \\
\text { de la sortie (croquis) } \\
\text { Cartographie sensible }\end{array}$ \\
\hline & $\begin{array}{l}\text { 2015, sortie-jeu dans le lycée (classe de } \\
\text { Première ES). }\end{array}$ & $\begin{array}{l}\text { Observation participante } \\
\text { Données prélevées par les } \\
\text { élèves } \\
\text { Recueil des } \\
\text { représentations à la fin } \\
\text { de la sortie (croquis) } \\
\text { Cartographie sensible }\end{array}$ \\
\hline & $\begin{array}{l}2018 \text { sortie-connectée dans les anciens } \\
\text { terrains Renault (classe de Première ES). }\end{array}$ & 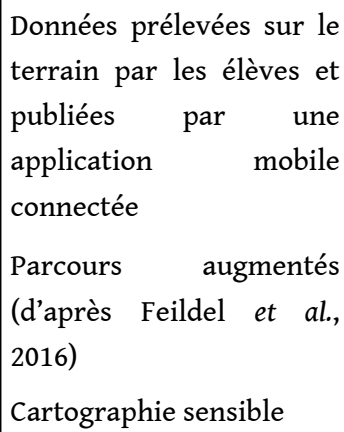 \\
\hline
\end{tabular}

\section{La sortie sensible: une pratique singulière, à la marge des pratiques ordinaires}

3 La sortie sensible est une pratique scolaire doublement originale. D'abord, parce que c'est une sortie et qu'à ce titre, en privilégiant une géographie "de plein vent» (Chevalier, 2007), elle se démarque des pratiques de classe ordinaires. Celles-ci privilégient une géographie «d'atelier » dans laquelle les documents, sélectionnés par l'enseignant et analysés entre les murs de la classe se substituent au réel. Les 
recherches de la géographie savante y sont présentées sous la forme de résultats, gommant toutes traces du processus de recherche scientifique, tout comme les débats dont ces résultats font l'objet (Audigier, 1993). Cela aboutit à une perception de l'espace par les élèves comme un donné davantage qu'un construit (Considère et Liénart, 2016, p. 275). La sortie de terrain a été réintroduite en 2008 dans les programmes de collège (BO spécial, $\mathrm{n}^{\circ}$ 6, 28 août 2008), et en 2010 dans ceux de lycée (BO spécial, $\mathrm{n}^{\circ} 9,30$ septembre 2010), en lien avec l'étude de "mon espace proche ", de l'habiter en classe de Sixième et des territoires de proximité en classe de Première, la sortie de terrain n'est plus mentionnée dans les nouveaux programmes de collège (BO spécial, $\mathrm{n}^{\circ} 11,26$ novembre 2015), l'étude de l'espace proche s'étant déplacée au primaire (classe de CM1). Il faut se tourner vers les documents d'accompagnement au programme (Eduscol) pour la retrouver associée à l'étude du local et de la prospective : en Sixième, des parcours sur le terrain sont recommandés pour l'étude de la ville de demain, en Cinquième une "visite de terrain » est proposée pour le chapitre sur les risques tandis qu'en Troisième, la possibilité d'une "enquête associant terrain et travail sur des documents » est évoquée pour le chapitre sur l'aménagement. Dans les programmes de lycée le contact avec le terrain est mentionné comme une des finalités de l'enseignement de la géographie. Les territoires de proximité sont étudiés dans le chapitre conclusif de terminale (BO spécial, $\mathrm{n}^{\circ} 8,25$ juillet 2019).

La sortie sensible se distingue par ailleurs des sorties de terrain telles qu'elles sont menées - lorsqu'elles ont lieu - dans le champ scolaire. Majoritairement, telles que j'ai $\mathrm{pu}$ les observer (corpus 1), elles exportent hors les murs les pratiques ordinaires; en cela, elles peuvent être caractérisées de sorties scolaires ordinaires. Toutes fonctionnent, en effet, sous la forme de questions fermées (une seule réponse est possible), conçues par l'enseignant (ou le médiateur à qui est confiée la sortie). La part laissée aux imprévus est réduite. Les évènements qui modifient l'environnement entre le moment où la sortie est conçue et celui où elle a lieu, ou lorsqu'elle a lieu (les intempéries, un chantier), peuvent empêcher tout ou partie de sa réalisation. Leurs modalités varient selon des dispositifs plus ou moins ludiques et donnant plus ou moins d'autonomie aux élèves (tableau 2).

Tableau 2. Typologie des sorties scolaires ordinaires sur le terrain

\begin{tabular}{|c|c|c|c|}
\hline & $\begin{array}{l}\text { Modalité } \\
\text { pédagogique }\end{array}$ & Nature du parcours & $\begin{array}{l}\text { Places du professeur et des } \\
\text { élèves }\end{array}$ \\
\hline $\begin{array}{l}\text { Sortie- } \\
\text { conférence }\end{array}$ & $\begin{array}{l}\text { Cours magistralo- } \\
\text { dialogué, proche des } \\
\text { pratiques de classe } \\
\text { ordinaires. }\end{array}$ & $\begin{array}{l}\text { Itinéraire, d'un point } \\
\text { A à un point B puis } C \text {, } \\
\text { etc. }\end{array}$ & $\begin{array}{l}\text { Le professeur (ou le } \\
\text { conférencier) parle, les élèves } \\
\text { écoutent et prennent des notes. } \\
\text { Les élèves sont invités à } \\
\text { prendre des notes. Les } \\
\text { bavardages sont considérés } \\
\text { comme une gêne. }\end{array}$ \\
\hline $\begin{array}{l}\text { Sortie } \\
\text { questionnaire }\end{array}$ & $\begin{array}{l}\text { Mise en activité des } \\
\text { élèves, assez proche } \\
\text { des pratiques de } \\
\text { classes ordinaires. }\end{array}$ & $\begin{array}{l}\text { Itinéraire, d'un point } \\
A \text { à un point } B \text { puis } C \text {, } \\
\text { etc. }\end{array}$ & $\begin{array}{l}\text { Par groupe de deux ou trois, les } \\
\text { élèves sont invités à retrouver } \\
\text { différentes informations dans } \\
\text { l'espace, formulées sous la } \\
\text { forme de questions fermées. }\end{array}$ \\
\hline
\end{tabular}




\begin{tabular}{|c|c|c|c|}
\hline $\begin{array}{l}\text { Sortie jeu de } \\
\text { piste/chasse au } \\
\text { trésor }\end{array}$ & $\begin{array}{l}\text { Mise en activité des } \\
\text { élèves sous la forme } \\
\text { d'une investigation et } \\
\text { par le recours à des } \\
\text { modalités ludiques. }\end{array}$ & $\begin{array}{l}\text { Parcours libre: } \\
\text { l'espace est une } \\
\text { surface } \quad \text { délimitée, } \\
\text { dans laquelle les } \\
\text { élèves sont libres de } \\
\text { se rendre où ils } \\
\text { veulent. }\end{array}$ & $\begin{array}{l}\text { Sous la forme de questions } \\
\text { fermées, les élèves sont invités } \\
\text { à retrouver différents indices } \\
\text { identifiés par leur professeur. }\end{array}$ \\
\hline
\end{tabular}

5 La sortie sensible, quant à elle, se caractérise par la mobilisation explicite d'une dimension sensible. Elle ne se réduit pas cependant à la mobilisation des cinq sens (la vue, l'odorat, le toucher, l'ouïe voire le goût), qui en ferait une simple lecture "augmentée » de paysage, mais elle inclut également le recours à l'imagination (comment était ce paysage il y a mille ans, comment il sera dans mille ans, quelle en serait ma perception si j'étais un géant, une fourmi, etc.), à la déambulation. Elle inclut, enfin, les évènements produits par notre environnement ou par notre interaction avec lui : la pluie, une voiture qui passe et éclabousse, le chantier qui couvre le son de la voix...

6 Ainsi définie, la sortie sensible se différencie des autres sorties scolaires sur le terrain. Ce n'est cependant pas une modalité pédagogique nouvelle. Sous ce nom de «sortie sensible » (la terminologie a été introduite dans le champ scolaire dès 2014 par Médéric Briand dans le cadre d'une thèse en didactique de la géographie consacrée à l'école primaire), ou sous d'autres terminologies, elle a déjà fait l'objet d'expérimentations dans le champ de la pratique, à la marge des pratiques ordinaires. Dans leur article consacré aux "parcours iconographiques ", Anne-Laure Le Guern et Jean-François Thémines (2011) ont retracé l'épistémologie de ces pratiques, comme les classespromenades, pratiquées par Célestin Freinet dès 1922. Le parcours iconographique, dont ils décrivent le dispositif et le fonctionnement, s'apparente également à la sortie sensible telle qu'elle vient d'être définie, il en constitue une catégorie particulière, la sortie sensible « relevé » qui sera définie dans la deuxième partie.

7 Par ailleurs, elle peut être rapprochée de certaines pratiques de terrain menées en géographie, et plus particulièrement en géographie de l'art, comme les parcours augmentés (Feildel et al., 2016) ou les expérimentretiens (Olmedo, 2017). Ces pratiques réinterrogent, depuis le champ scientifique, celles des artistes-marcheurs, initiées aux États-Unis dans les années 1970 par les artistes du land art, comme les artistics walks d'Amish Fulton. Elles sont très présentes dans le champ artistique contemporain français, sous des formes participatives, comme les promenades floues décrites par l'artiste Mathias Poisson sur son site ${ }^{2}$, ainsi nommées car elles se font avec des lunettes floues, ou les propositions de l'agence Touriste qui se définit ainsi : «L'Agence Touriste est une agence de promenade expérimentale qui propose d'inventer et de pratiquer un tourisme de travers pour explorer des territoires méconnus (quartiers sans monuments, périphéries de villes, lieux intermédiaires). Elle organise des dérives (visites guidées, performances, ateliers) et fabrique des traces de voyage avec les moyens du bord (cartes subjectives, textes, expositions, performances...) $»^{3}$.

8 Elle se rapproche également, dans le champ de la médiation culturelle, des visites sensibles organisées par certains organismes en charge de la médiation de l'architecture, de l'urbanisme et de l'environnement, et à destination d'un jeune public 
ou d'adultes, comme les CAUE (Conseil d'Architecture, d'Urbanisme et d'Environnement).

9 À la différence de ces pratiques artistiques, scientifiques ou de médiation, la sortie sensible scolaire ne vise pas tant la production d'un savoir scientifique nouveau, la concrétisation d'une pensée sous la forme d'une œuvre artistique ou la médiation d'un savoir dans un contexte extra-scolaire que l'apprentissage par les élèves de la géographie. C'est sur ce point que nous allons nous pencher à présent. En effet, après avoir défini ce qui caractérise la sortie sensible et l'avoir positionnée dans le champ des pratiques scolaires comme une pratique singulière, à la marge des pratiques ordinaires, et par rapport aux champs artistiques et scientifiques dont elle se rapproche, sans s'y confondre, dans sa spécificité de pratique «scolaire ", il nous faut à présent examiner les savoirs qu'elle met en jeu et la manière dont elle les met en jeu. Je m'appuierai pour cela sur la description de dispositifs que j'ai conçus et expérimentés dans le champ de ma pratique d'enseignante.

\section{Les modalités de la sortie sensible}

Enseignante d'histoire-géographie en lycée, j'ai observé (corpus 2) et expérimenté avec des classes de Première ES (corpus 3) (voir tableau 1) différents dispositifs de sorties sensibles, en tant que praticienne (dès 2011), puis praticienne-chercheure (à partir de 2012). "Bricolages", au sens de Lévi-Strauss $(1962)^{4}$, ces dispositifs sont des réponses pratiques apportées à des questionnements survenus dans un contexte donné, celui de nouvelles instructions officielles, par exemple, et sur un terrain local, l'espace proche de mes élèves (l'espace du lycée ou un espace situé à proximité, les anciens terrains Renault). Tous avaient le même objectif : permettre aux élèves, au cours d'une sortie sur un territoire situé à proximité du lycée, de porter une attention particulière à leur environnement, de construire de manière collective des savoirs d'expérience sur l'espace, d'y intégrer leurs savoirs d'expérience individuels, puis de les articuler aux savoirs formels de la géographie scolaire. Il s'agissait ainsi de mettre en œuvre une géographie expérientielle, telle qu'elle est définie par le groupe Pensée Spatiale de l'IREM ${ }^{5}$. De manière itérative, par des va-et-vient entre ma pratique et la recherche, j'ai expérimenté successivement quatre modalités différentes, dont j'ai identifié les variables, ajusté les paramètres (figure 1). Je les présenterai ici tour à tour.

Figure 1. Méthodologie de la recherche

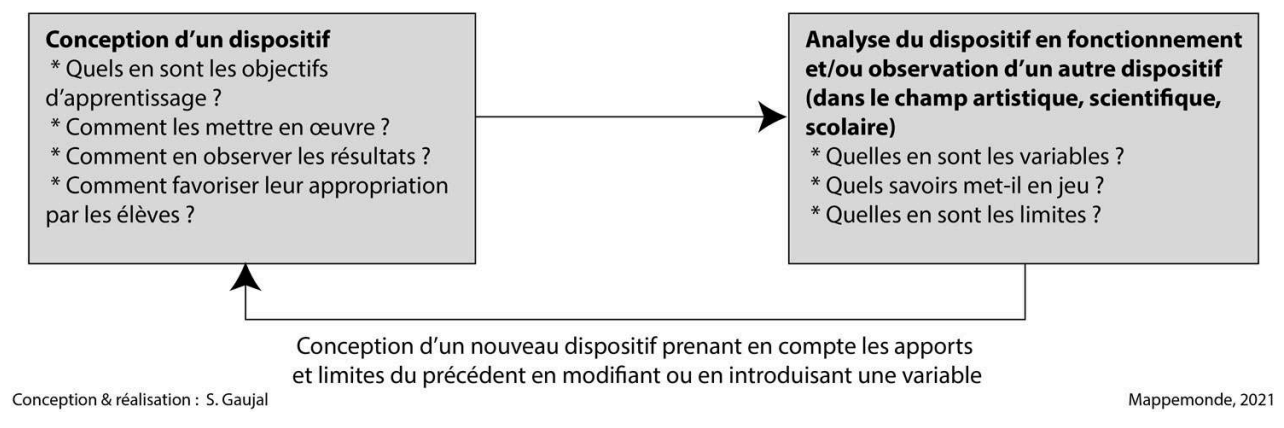

Réalisation : S. Gaujal 


\section{Dispositif $n^{\circ} 1$ (2011) : la sortie « relevé »}

11 La première sortie, que j'identifie a posteriori comme une sortie sensible, a été conçue en 2011, alors que venait d'être mise au programme de Première l'étude de l'aménagement des territoires de proximité (BO spécial, $n^{\circ} 9,30$ septembre 2010), qui offrait l'opportunité d'une sortie de terrain, mais qui présentait aussi le risque qu'ils soient étudiés " comme n'importe quel ailleurs» (Gaujal, Leininger et Vergnolle, 2017). En effet, la difficulté d'une telle étude est d'articuler le savoir d'expérience des élèves (savoir souvent supposé plutôt qu'effectif, car territoire proche ne signifie pas nécessairement territoire connu des élèves) avec le savoir expert de l'enseignant (quoique souvent peu informé dans le détail des aménagements du quartier dans lequel il enseigne, mais n'habite pas). Pour surmonter cet obstacle, j'ai proposé de mettre en place un concours de photographie mettant en compétition deux classes de Première ES. Il s'agissait ainsi, par une consigne motivante, à la fois d'inciter les élèves à observer (voire à découvrir) leur espace proche et ses aménagements et de formaliser leurs observations. La consigne était de produire une photographie originale, de manière individuelle ou par groupe, accompagnée d'un titre et d'une note d'intention, sur le thème de l'aménagement des anciens terrains Renault.

12 Afin d'analyser les apprentissages que ce concours permet de mettre en œuvre, je me suis intéressée à la portion d'espace photographiée (type d'espace, format, point de vue), aux notes d'intention (expression d'un point de vue, explication de la manière dont est construite la photographie, énonciation d'un savoir factuel), à la distance parcourue par les élèves par rapport au lycée que j'ai déduite des objets qu'ils ont photographiés. Plus généralement, j'ai été attentive aux indices qui révélaient la manière dont les élèves ont interagi avec l'environnement au cours de la sortie qui leur était proposée. Comme critères de réussite de l'exercice, je me suis également basée sur des critères subjectifs, comme, par exemple, le plaisir que nous avons eu avec ma collègue Ariane Jourdan, co-organisatrice du concours, à découvrir certaines productions et la manière dont nous avons progressivement ajusté notre évaluation ${ }^{6}$. Enfin, j'ai demandé l'année suivante à quelques élèves de me confier l'ensemble des photographies qu'ils ont prises le jour de leur sortie et de les commenter. J'ai ainsi pu reconstituer leur parcours iconographique.

13 Je suis ainsi arrivée à plusieurs conclusions : tout d'abord, sur un plan quantitatif, le nombre d'élèves qui ont interagi avec l'espace a été relativement limité. La grande majorité d'entre eux ne se sont pas emparés de l'exercice, ce qui peut s'observer de plusieurs manières. Certains n'ont pas fait la visite. Si tous, en effet, ont bien rendu une production, certains ont présenté une photographie prise sur Internet. Ces élèves ne se sont donc pas emparés de l'exercice, le transformant en une tâche scolaire dont il faut s'acquitter, quitte à en transgresser les règles (qui exigeaient une photographie originale). D'autres se sont rendus sur le terrain. Mais les objets/portions d'espace qu'ils ont photographié montrent qu'ils ne sont pas entrés dans une logique d'exploration, se cantonnant à des espaces situés à proximité de l'établissement scolaire (figure $2^{7}$ ). 
Figure 2. Sortie-relevé, 2011, le parcours des élèves

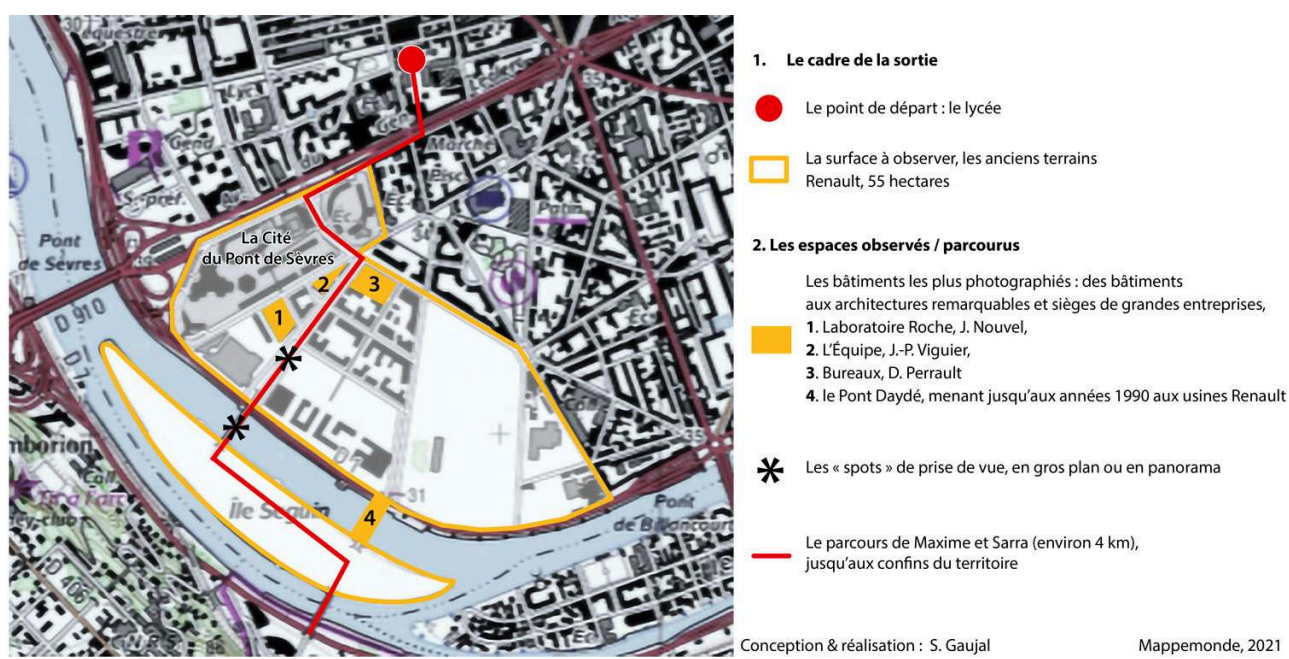

Réalisation : S. Gaujal

$1453 \%$ de leurs photographies sont des panoramas, reproduisant des paysages qui rappellent ceux des manuels scolaires décrits par Didier Mendibil (1999), ou des gros plans de bâtiments. 58 \% des objets photographiés sont la Tour Horizon de Jean Nouvel (achevée en 2011), nouveau repère du quartier et photographiée sous tous les angles, et le Pont Daydé, emprunté par près de 10000 ouvriers chaque jour dans les années 1960 et symbolisant le passé (figure 3). Les notes d'intention qui les accompagnent sont descriptives, reprenant, parfois au mot près, des notices Wikipédia sur le quartier.

Figure 3. La tour Jean Nouvel et le pont Daydé sous toutes les coutures
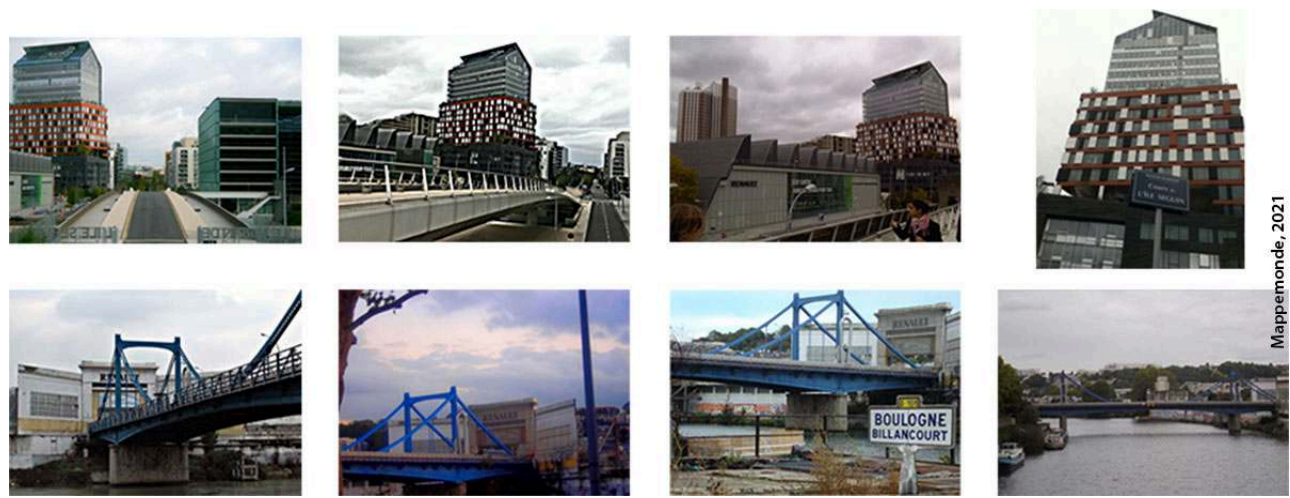

Réalisation : S. Gaujal, corpus de S. Gaujal

Quelques-uns, enfin, ont proposé des approches plus complexes. C'est le cas de Maxime et Sarra (lauréats de notre concours), dont j'ai pu reconstituer le parcours à partir des photographies qu'ils ont prises ce jour-là et de l'entretien que j'ai eu avec Maxime l'année suivante. Ce parcours les a conduits, en compagnie d'un camarade d'une autre classe, au moment du déjeuner, sur une distance d'environ $4 \mathrm{kms}$, aux confins du territoire observé (figure 2). Au cours de cette sortie, ils ont joué (figure 4a); ils sont allés dans des endroits interdits (figure $\mathbf{4 b}$ ); ils se sont mis en scène, imitant un ouvrier (figure 4c, figure $\mathbf{4 d}$ ) ; et ils ont mis en scène la photographie qu'ils ont choisi de présenter au concours (figure 4e), la retouchant en partie (figure 4f) (peu visible sur la reproduction présentée ici) pour produire un contraste entre le passé (un ouvrier 
mis en scène, en noir et blanc) et le présent (un paysage présentant au premier plan l'île Seguin et à l'arrière-plan les bâtiments construits/en construction du Trapèze). Au total, ils ont passé 44 minutes sur lîle, au cours desquelles ils ont pris 63 photographies.

Figure 4. Maxime et Sarra sur le terrain, 2011
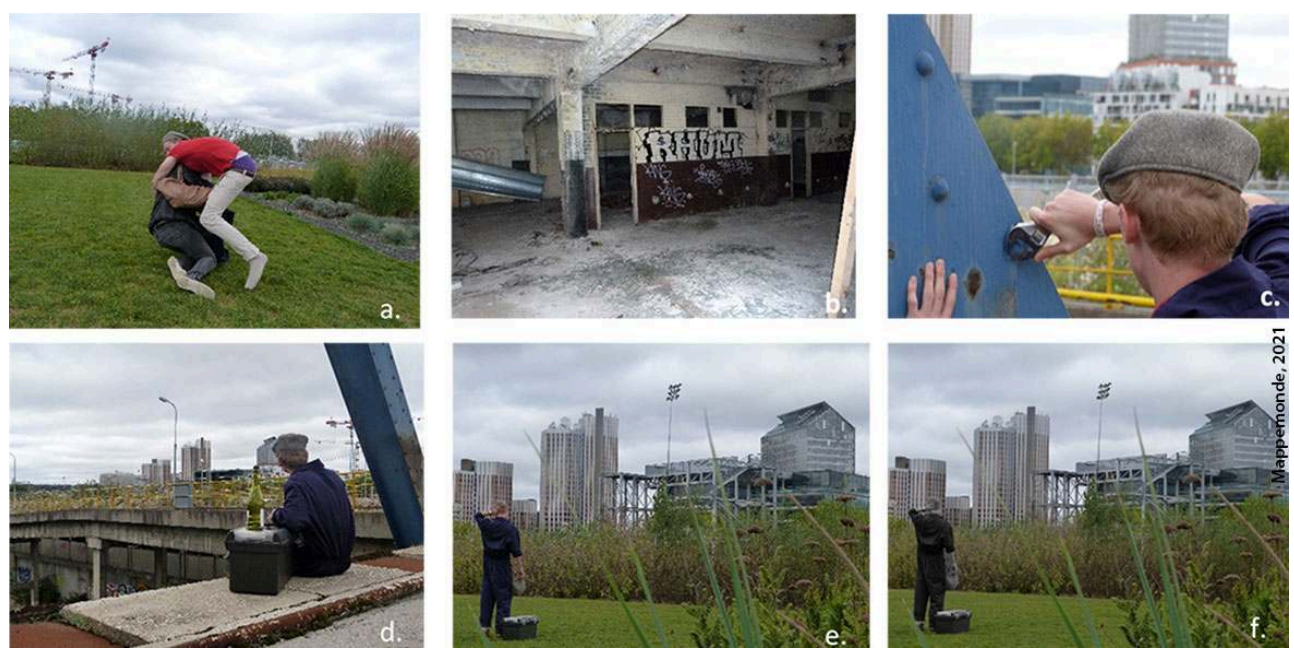

Réalisation : S. Gaujal, corpus de S. Gaujal

Trois types de rapport à l'espace ont ainsi été mis en jeu: certains ont agi SANS l'espace, d'autres se sont questionnés SUR l'espace, d'autres enfin, plus rares, comme Maxime et Sarra ont interagi AVEC l'espace ${ }^{8}$. Les moyens de cette interaction ont été l'exploration (découverte d'endroits inconnus, voire interdits, sur le principe de l'URBEX'), le jeu (lutte dans l'herbe) et le recours à l'imagination (mise en scène d'un ouvrier des années 1960). Ils ont nourri la conception des dispositifs qui ont suivi. Restait, néanmoins, à trouver une modalité permettant d'engager tous les élèves dans cette démarche, et pas quelques-uns seulement. Le dispositif conçu par le CAUE pour une de mes classes, l'année suivante, m'a permis d'avancer dans cette direction.

\section{Dispositif n² (2012) : la sortie sensible « dérive »}

17 La consigne donnée aux élèves lors de cette nouvelle sortie ${ }^{10}$ reprenait et systématisait les modalités déjà observées lors de la sortie de Maxime et Sarra : l'imagination et l'exploration. Elle mobilise d'autres modalités recourant plus explicitement aux cinq sens : la vue (par la privation de l'ouïe) ou l'ouïe (par la privation de la vue). Réalisée sur le temps scolaire, elle permet une expérience collective tout en laissant les élèves libres d'aller où ils le souhaitent sur le terrain, par groupes de deux ou trois, à la manière d'une "dérive » (Debord, 1958), dans les limites néanmoins de l'espace exploré, l'île Seguin (12 hectares).

18 Les observations faites au cours de cette sortie montrent comment les élèves s'en sont emparés. Par exemple, sur la photographie ci-dessous (figure 5), on voit une élève juchée sur les épaules d'un camarade, s'imaginant être un géant. 
Figure 5. Esther sur les épaules de Valentin, jouant à être un géant, octobre 2012

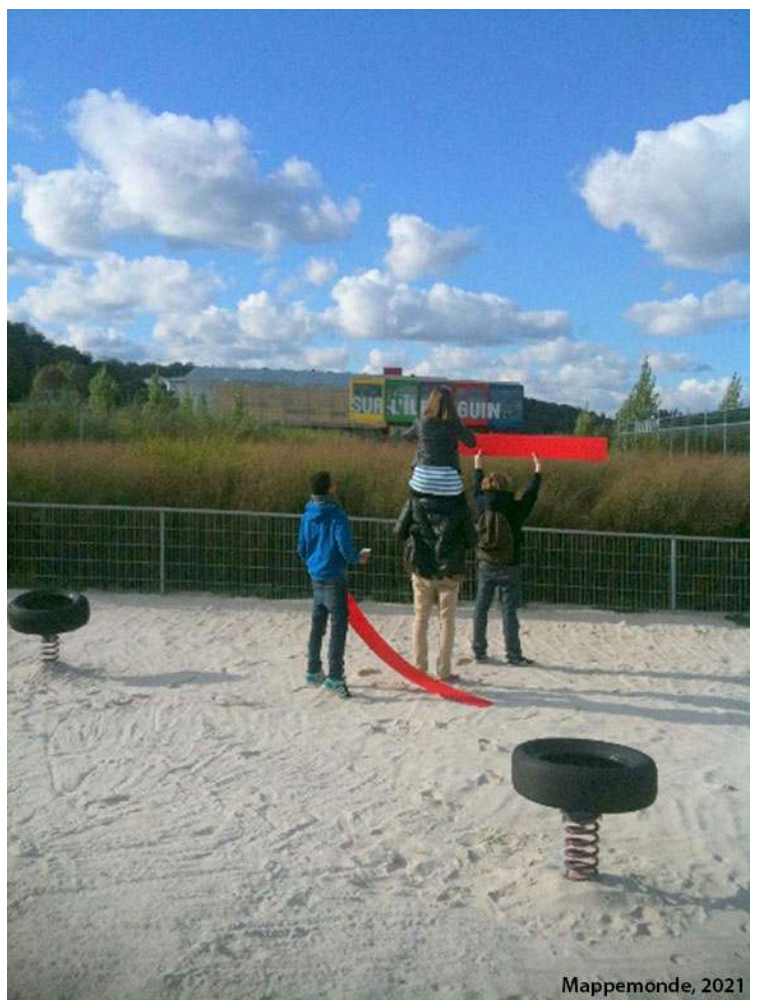

Source : corpus de S. Gaujal

19 Néanmoins, tous ne se sont pas investis de la même manière : certains élèves ont peu bougé et exploré l'espace, restant à proximité du pont où nous les avions quittés (figure 6). D'autres ont réalisé un parcours plus riche, se dirigeant soit vers la pointe aval, comme le groupe 1 , soit vers la pointe amont de l'île, comme le groupe 2 .

Figure 6. Sortie « dérive », le parcours des élèves, 2012

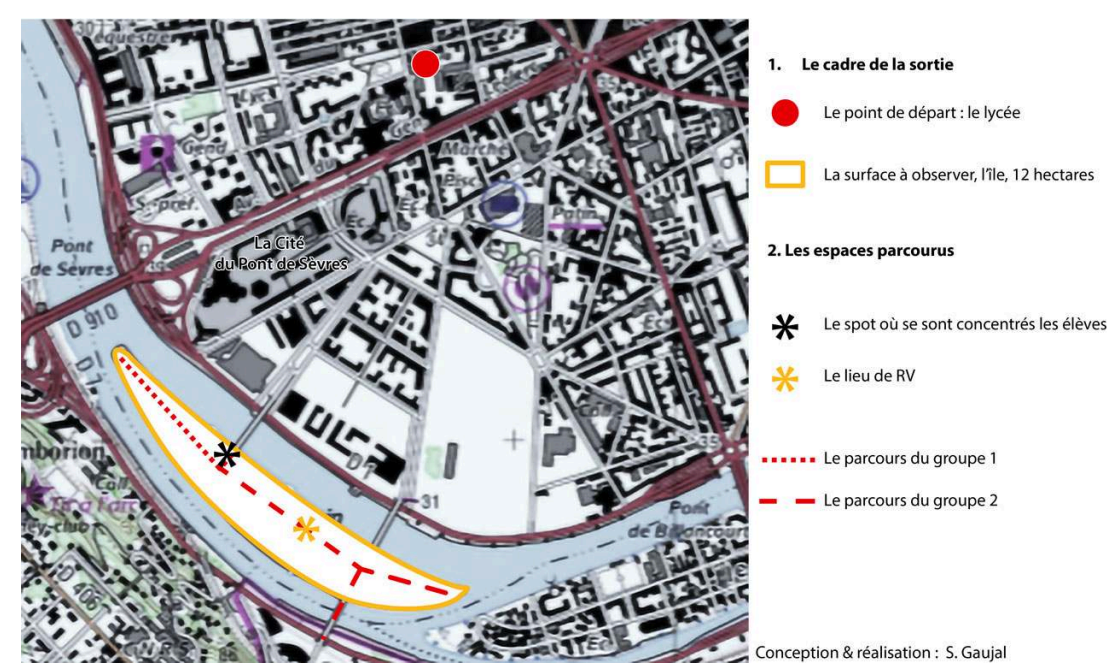

Réalisation : S. Gaujal

20 Par ailleurs, les productions photographiques qu'ils ont rendues à l'issue de cette sortie (photographies prises sur le temps de la sortie ou hors du temps scolaire) révèlent une 
répartition assez similaire des élèves dans leur rapport au savoir, sans toutefois qu'il y ait eu cette de productions « sans » l'espace.

\section{Dispositif $n^{\circ} 3(2014,2015)$ : la sortie « jeu ».}

Le nouveau dispositif que j'ai testé en 2014, puis à nouveau en 2015 avait pour objectif d'impliquer l'ensemble du groupe classe, et pas seulement les élèves les plus volontaires. C'est pourquoi je l'ai conçu sous la forme d'une visite guidée, alternant des jeux d'observation par deux ou collectifs. Ils sont schématisés sur la figure 7 : jeu d'écoute, consistant à faire une minute de silence sur le pont de Sèvres $\left(n^{\circ} 1\right)$, puis marche de la classe en silence vers la cité du Pont de Sèvres ( $\left.n^{\circ} 2\right)$, à laquelle les élèves accèdent deux par deux, l'un les yeux bandés et l'autre le guidant. Ces exercices ont été ensuite répétés à différents endroits, sous la conduite d'une élève. Nous avons également procédé à d'autres jeux : jeux d'acoustique (par exemple, crier «écho » sur la place haute des Alpes [ $\left.\mathrm{n}^{\circ} 3\right]$ ), cache-cache improvisé dans la cité.

Figure 7. La sortie « jeu », le parcours de la classe, 2014

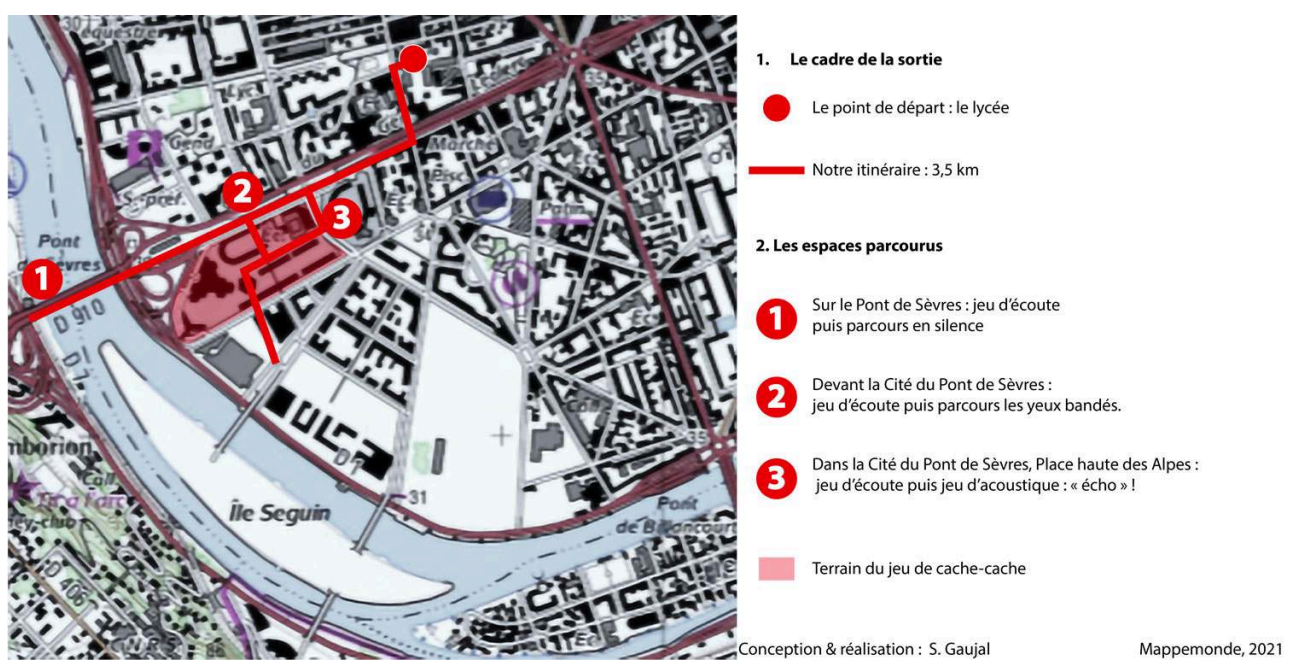

Réalisation : S. Gaujal

En termes d'investissement des élèves, l'objectif poursuivi semble atteint. Les enregistrements réalisés au cours de cette sortie sont ponctués de rires et d'exclamations étonnées. Une élève dira même : «j'ai l'impression d'être retombée en enfance ». Cependant, en réduisant la part d'autonomie des élèves, elle nécessite, de retour en classe, un étayage plus important, expliquant en quoi les jeux auxquels la classe a participé sont révélateurs de règles d'organisation de l'espace (Gaujal, 2020). En quoi, par exemple, la consigne "écho " montre-t-elle l'acoustique très particulière de cette partie de la cité, dans laquelle les immeubles sont disposés en amphithéâtre, avec une forte réverbération des sons? En quoi le jeu du «colin-maillard» permet-il de mieux appréhender le dénivelé de la cité et son urbanisme de dalle ? Ou en quoi le jeu de cache-cache montre-t-il sa dimension labyrinthique?

Selon les mêmes modalités, j'ai renouvelé ce dispositif l'année suivante sur un autre terrain, le lycée, le plan Vigipirate nous interdisant toutes sorties à l'extérieur. 


\section{Dispositif $n^{\circ} 4:$ la sortie « connectée »}

La sortie « connectée » est le dernier dispositif que j'ai conçu (2018), et inédit jusqu'ici. Trois nouvelles modalités y sont introduites. Tout d'abord, le recours à une application mobile qui permet une communication à distance entre les élèves (mobiles) et les professeurs accompagnateurs (un professeur documentaliste, une professeure d'EPS et moi-même) positionnés à un point fixe sur l'île Seguin. 6 groupes (6 élèves par groupe) se sont ainsi déplacés sur trois terrains différents, comme le montre la figure 8 : la cité du Pont de Sèvres, le Trapèze, l'île Seguin. La suite de la sortie s'est réalisée avec toute la classe, selon les modalités de la sortie « jeu » décrite ci-dessus.

Figure 8. Sortie « connectée », 2018, le parcours des élèves

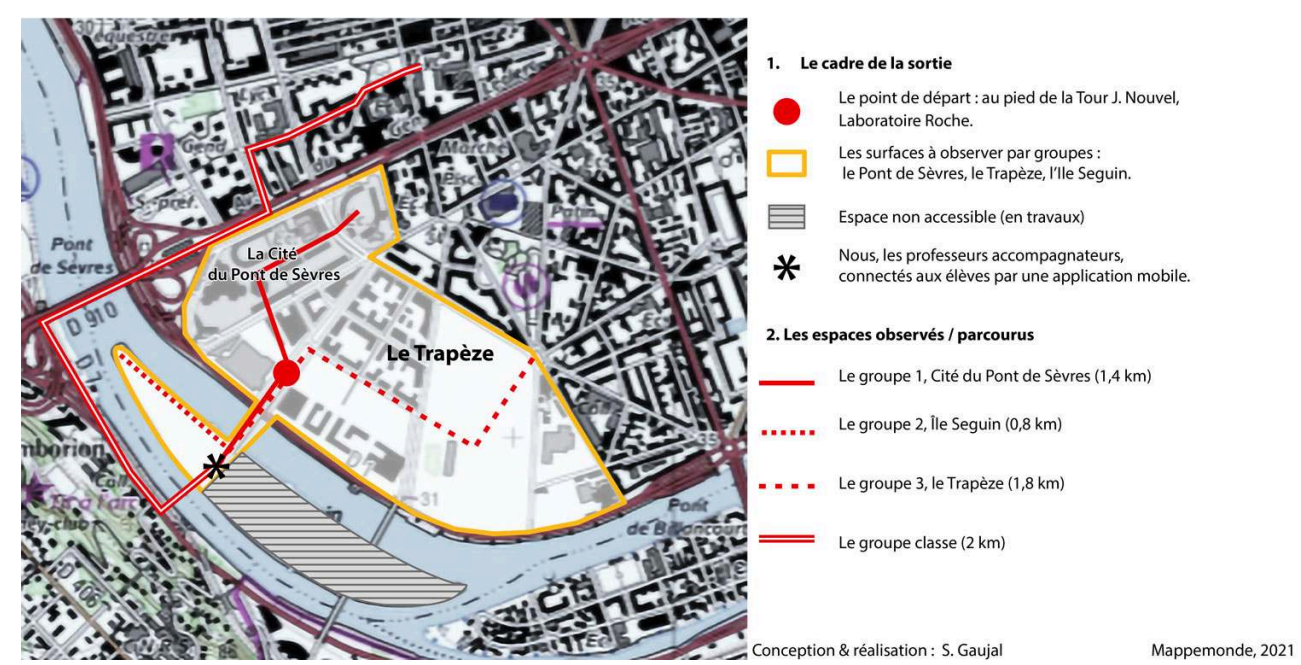

Réalisation : S. Gaujal

Différentes consignes ont été proposées via l'application mobile comme, par exemple : «à partir d'une photographie ancienne, retrouver un lieu et le reprendre en photo sous le même angle "; "chercher la boule à facette (la Seine Musicale sur l'île Seguin) et danser devant »; « le point de rendez-vous porte le même nom qu'un conte d'Alphonse Daudet, où suis-je ? (île Seguin)» (figure 9). 
Figure 9. Capture d'écran, sortie « connectée », octobre 2018



Source : corpus de S. Gaujal

Par ailleurs, une nouvelle consigne a été introduite, consistant à inviter les élèves à partir à la rencontre des habitants, en enregistrant leurs témoignages.

27 Enfin, au retour de la sortie, j'ai demandé aux élèves de réaliser des "parcours augmentés » analogues à ceux décrits par Benoît Feildel et al. (2016), et qui consistent à tracer, sur une même feuille, le parcours réalisé, la courbe des sons entendus, les ressentis et les objets rencontrés. Ce dispositif permet, selon Gaujal, «entre art et géographie", "une conscientisation et une remise en question des habitudes perceptives ", en associant la marche avec une restitution narrative. Un exemple en est présenté ci-dessous (figure 10). 
Figure 10. Un exemple de parcours augmenté. Sortie « connectée », 2018



Source : corpus de S. Gaujal

Outre les opérations de "conscientisation » qu'il avait pour objectif de favoriser, ce corpus de parcours augmentés ( 35 au total) permet d'identifier les ressentis des élèves. Cela aboutit au nuage de mots ci-dessous (figure 11).

Figure 11. Les ressentis des élèves à l'issue de la sortie " connectée »

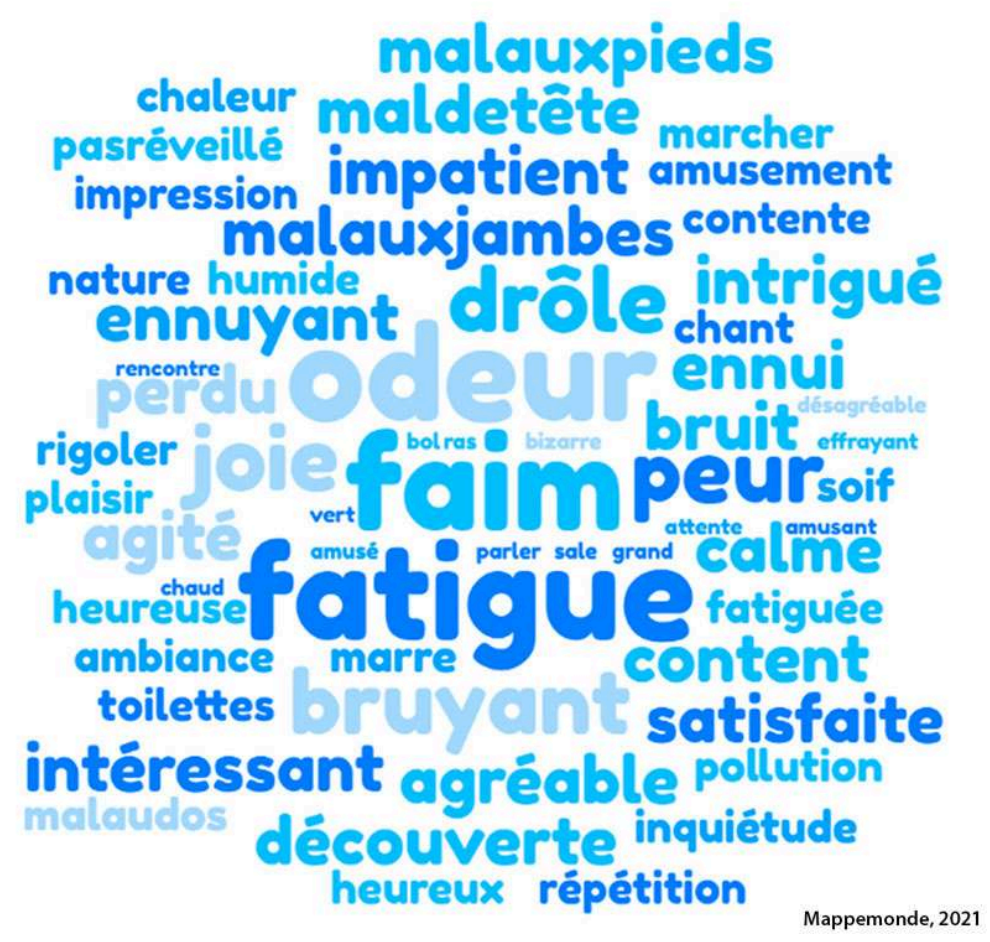

Réalisation : S. Gaujal 
Plusieurs types de sensations se détachent: des sensations liées à la distance, qui s'exprime par de la fatigue (entre 3 et $4 \mathrm{kms}$ ont été parcourus) ("fatigue», " épuisement ", « marre de marcher ", " mal aux pieds ", « mal aux jambes ", « mal au dos ", «mal de tête »), et qui ont conduit un élève à ne pas participer à la sortie, au motif, a-t-il expliqué " que c'était trop loin »; cas d'un élève qui, comme pour la sortie « relevé », a choisi de faire « sans » l'espace. Troublés dans leur routine, quasiment tous sont contents de rentrer («contente de retrouver mes amis» «joie de retourner au lycée » " heureux d'avoir terminé »), même si l'un d'entre eux confie : "sentiment de répétition de fréquenter le même établissement et ras-le-bol général de la routine des cours ». On note également des sensations liées spécifiquement au corps de ces adolescents de 16-17 ans : tous éprouvent un sentiment de faim que viennent réveiller les odeurs de boulangerie, ne se sentent "pas réveillés", "pas en forme ». D’autres sensations encore sont liées aux lieux traversés : «bruyant ", " chaud », "peur d'être renversé par un camion", "sensation de bruit intense sur le Pont de Sèvres avec les voitures qui passent »; « odeur désagréable »; « odeur de feuille mouillée »; « odeur de nature »; "sensation de fraîcheur à côté de la Seine: le soleil était bon", «bruit ambiant de la ville», "forte densité de voiture ", «sentiment de tranquillité dans le parc "; « agréable cependant pas assez d'espaces accessibles pour les passants donc pas assez libres ». Le dispositif lui-même est commenté : « excité », " ennuyeux », « drôle », " amusant ", « intéressant ", "j'aime bien marcher ", " gênance ", "flemme », " plaisir de chercher des endroits où trouver l'énigme ou d'interroger des passants et de réussir ». Enfin, les élèves apprécient également les relations que cela contribue à créer au sein de la classe : «j'ai passé un bon moment avec la classe »; « confiance dans mon ami William ", "plaisir à faire le travail et à chercher ce qu'on devait trouver avec le groupe », « sympa, tout le monde rigolait ensemble».

\section{Conclusion}

La série de dispositifs présentée ici permet, au-delà de la singularité propre à chacun, de dégager quelques principes généraux. Ainsi, différents exercices permettent de porter une attention particulière à son environnement, de manière autonome, guidée ou semi-guidée à distance : le recours, seul, par deux ou en groupe à l'imagination, à des jeux d'observation par la privation d'un sens ou l'exacerbation d'un autre, à l'exploration et à la rencontre avec des habitants. Les résultats varient selon les dispositifs : plus les élèves ont d'autonomie, moins les échanges à l'intérieur du groupe classe sont nombreux, fragmentant les apprentissages à de petits groupes plus ou moins investis (sans ou dans l'espace) et plus ou moins engagés dans l'exercice (sur ou avec l'espace). Au contraire, plus les élèves sont guidés, plus les échanges à l'intérieur du groupe classe sont nombreux, mais plus ils nécessitent un étayage de la part de l'enseignant à l'issue de la sortie, au risque, sinon, que seule la dimension récréative de la sortie soit perçue, sans générer d'apprentissage. La sortie "connectée » est un compromis entre ces deux pôles. Elle pose, cependant, la question de l'usage des mobiles et de sa transposition avec des élèves plus jeunes. Toutes, enfin, interrogent la compréhension que les élèves ont de tels dispositifs. Les commentaires suivants, recueillis à l'issue des projets de cartographie sensible en 2016 et 2018, témoignent d'une appropriation variée: pour certains, le projet se justifie "parce qu'ici tout est là »; " parce qu'on a appris à mettre du grand dans du petit », témoignant de leur 
compréhension des finalités intellectuelles du travail entrepris. Pour d'autres, les finalités restent résolument culturelles, fidèles à la représentation d'une discipline de mémorisation : «j'ai appris qu'il y a le pouce de César à la Seine Musicale ». Pour un autre, enfin (celui qui n'est pas venu à la sortie en 2018), "ça ne sert à rien, car je connais déjà »; le même dira cependant quelques lignes plus loin : « la géographie, ça sert à se poser des questions que quotidiennement on ne se pose pas parce qu'on trouve ça normal ». Analyser finement cette appropriation, en prenant en compte les variables du capital scolaire, du sexe, de l'âge, de l'arrivée plus ou moins récente dans le quartier, ainsi que les implicites scolaires et les sauts cognitifs engendrés par les dispositifs euxmêmes, est l'objectif que s'est fixé le groupe Pensée Spatiale pour son prochain cycle de recherche.

\section{BIBLIOGRAPHIE}

AUDIGIER F. (1993). Les représentations que les élèves ont de l'histoire et de la géographie. À la recherche des modèles disciplinaires, entre leur définition par l'institution et leur appropriation par les élèves. Thèse, Paris 7.

BRIAND M. (2014). La géographie scolaire au prisme des sorties : pour une approche sensible à l'école élémentaire. Thèse, Caen. En ligne : http://www.theses.fr/2014CAEN1027

CONSIDÈRE S., LIÉNART O. (2016). « Des acteurs en cours de géographie? » In J.-F. THÉMINES ET S. Doussot (dir.), Acteurs et Action. Perspectives en Didactiques de l'Histoire et de la géographie. Caen : Presses universitaires de Caen, p. 275 sqq.

DEBORD G. (1958). Internationale situationniste. $\mathrm{N}^{\circ}$ 1, juin 1958.

FEILDEL B., OLMEDO É., TROIN F., DEPEAU S., POISSON M., AUDAS N., JAULIN A. et DUPLAN K. (2016). « Parcours augmentés, une expérience sensible entre arts et sciences sociales ». Carnets de géographes, $\mathrm{n}^{\circ} 9$ (août). En ligne : https://doi.org/10.4000/cdg.721

GAUJAL S. (2016). Une géographie à l'école par la pratique artistique. Thèse, Université Paris 7 - Denis Diderot. En ligne : https://tel.archives-ouvertes.fr/tel-01582642/document

GAUJAL S. (2017a). «Au carrefour de l'histoire des arts et de la géographie : apprendre à lire la ville avec une classe de Première ». In A.-S. MOLINIÉ ET G. DI ROSA, Feux croisés sur les pratiques en histoire des arts. La didactique de l'histoire et de la géographie au miroir de l'histoire des arts, Paris :

L'Harmattan, p. 105-131.

GAUJAL S. (2017b). « La cartographie sensible participative : un autre rapport au savoir ». Diversité, $\mathrm{n}^{\circ} 189$.

GAUJAL S., VERGNOLLE-MAINAR C., LEININGER C. (2017). « La géographie scolaire et le territoire de proximité ». In P. CHAMPOLLION, Y. ALPE et A. BARTHES, Permanences et évolutions des relations complexes entre éducation et territoires, Londres : ISTE Éditions, p. 139-154.

GAUJAL S. (2020). « La ville comme terrain de jeu en géographie ». In M. PRÉVOT, É. MONIN et N. DOUAY, L'urbanisme, l'architecture et le jeu, Lille : Presses Universitaires du Septentrion, p. 43-54. 
LE GUERN A.-L., THÉMINES J.-F. (2011). « Des enfants iconographes de l'espace urbain : méthode du parcours iconographique. » Carnet de géographes, $n^{\circ}$ 3. En ligne : https://journals.openedition.org/ $\operatorname{cdg} /$

LÉVI-STRAUSS C. (1962). La pensée sauvage. Paris : Plon.

MENDIBIL D. (1999). « Essai d'iconologie géographique ». L'Espace géographique, vol. 28, nº 4, p. 327-36. En ligne : https://doi.org/10.3406/spgeo.1999.1276

OLMEDo É. (2012a). «Cartographier les interstices de la ville Mathias Poisson ». Strabic. En ligne : http://strabic.fr/Mathias-Poisson-Cartographier-les-interstices-de-la-ville

OLMÉDO E. (2012b). « Hendrik Sturm, l'infatigable marcheur-sculpteur d'espace ». Strabic. En ligne : http://strabic.fr/Hendrik-Sturm-l-infatigable

OLMEDo É. (2017). « L'expérimentretien comme méthode d'enquête. Cartographie sensible et terrains de recherche collaboratifs entre art et géographie ». Mappemonde, $\mathrm{n}^{\circ} 121$. En ligne : https://journals.openedition.org/mappemonde/3776

PIGAKI M., LEININGER-FRÉZAL C. (2014). « Enseigner les disparités socio-spatiales avec hyperatlas : le cas de l'Union européenne ». Didáctica Geográfica 1, n 15, p. 79-107.

THÉMINES J.-F. (2016). « La didactique de la géographie ». Revue française de pédagogie. Recherches en éducation, $\mathrm{n}^{\circ}$ 197, p. 99-136. En ligne : https://doi.org/10.4000/rfp.5171

VOLVEY A. (2012). Transitionnelles géographies: sur le terrain de la créativité artistique et scientifique. HDR, Université Lumière - Lyon II.

\section{NOTES}

1. Au sens défini par Jean-François Thémines qui distingue la «situation", "une notion faiblement construite " à la différence de celle de "dispositif », " que le didacticien, en position de formateur et/ou de chercheur, met en place pour expérimenter des pratiques alternatives » (2016, p. 115).

2. Mathias Poisson, «Promenades floues", http://poissom.free.fr/? browse=promenades\%20floues, dernière consultation le 13 janvier 2019.

3. Agence Touriste, page d'accueil, http://netable.org/?browse=l\%27Agence\%20Touriste, dernière consultation le 13 janvier 2019.

4. Soit une forme de "pensée sauvage ", une inventivité au quotidien, dans l'improvisation, qui n'hésite pas à détourner des outils existants pour les adapter au plus près de ses besoins. (Gaujal, 2016, p. 152 et sq.)

5. Constitué depuis 2015 autour d'une réflexion sur la «pensée spatiale », ce groupe est formé par une enseignante-chercheure (Caroline Leininger-Frézal), des enseignants du primaire (Xavier Leroux) et du secondaire (Pierre Colin, Florence Giry, Catherine Heitz, et moi-même).

6. Mail envoyé par Ariane Jourdan (AJ) le 14 octobre : «J'ai reçu la plupart des photos pour le concours de mes groupes de 1 ère ES. C'est pas mal! Cette idée de concours était très bienvenue ». Ma réponse (SG), 14 octobre: «Pour les photos moi aussi je suis contente. Les photos sont de qualité inégale mais c'est un très bon exercice, ils apprennent à regarder ». Et toujours le 14 octobre, $\mathrm{AJ}$ : «Ce qui m'ennuie davantage est la qualité des commentaires, très inégaux en longueur et en qualité même si tous ont fait l'effort de le rédiger. Je pense que je le prendrai en compte dans une petite note sur 5 (additionnée à d'autres petits travaux qu'ils ont faits), mais peut-être pour le jury vaut-il mieux laisser la photo parler d'elle même... Enfin, on réfléchira aux modalités. La semaine prochaine?». 
7. Comme l'ensemble de ceux proposés dans cet article, ce croquis a été réalisé à partir d'une carte IGN au $1 / 25000^{\circ}$.

8. Maria Pigaki et Caroline Leininger (2014) identifient trois types de rapport à l'espace : SUR l'espace, DANS et AVEC l'espace. Cette typologie a été reprise par notre groupe Pensée Spatiale.

9. URBEX : exploration urbaine définie par l'encyclopédie en ligne Wikipédia comme «une activité consistant à visiter des lieux construits par l'homme, abandonnés ou non, en général interdits, ou tout du moins cachés, ou difficiles d'accès. L'explorateur urbain est aussi appelé urbexer.»

10. «Explorez le lieu en vous laissant guider par votre ressenti, vos impressions : ce qui vous attire, vous repousse, vous pose question. Testez des points de vue ou des modes d'observation inhabituels : en vous couchant par terre, en marchant à reculons, en courant, en tournant sur soi-même à $360^{\circ}$, en se mettant des œillères ou quelque chose qui cadre le regard, en fermant les yeux ou en se bouchant les oreilles, en imaginant être un géant ou une fourmi... Projetez votre imaginaire sur le lieu : et si je visitais ce lieu la nuit à la lueur de la bougie? Et si je m'y retrouvais seul pendant $24 \mathrm{~h}$, où irais-je et que ferais-je ? Et si ce lieu devenait le décor d'un film, de quel genre serait-il et quel serait son scénario ? Et si je devais cacher un trésor ou laisser un objet familier dans cet espace? Projetez-vous dans le temps : selon vous comment était ce lieu il y a 1000 ans, 100 ans, 10 ans ? Et que sera-t-il dans 10 ans, 100 ans, 1000 ans ? À l'issue de cette visite sensible, donnez 5 mots qui expriment votre ressenti, vos impressions (subjectives et personnelles sur l'île Seguin). [Qui viennent compléter les 5 mots recueillis avant la sortie] ».

\section{RÉSUMÉS}

La sortie sensible est une pratique scolaire doublement originale. D'abord, parce qu'en privilégiant une géographie "de plein vent» (Chevalier, 2007), elle se démarque de la "géographie d'atelier » dans laquelle les documents, sélectionnés par l'enseignant et analysés entre les murs de la classe, se substituent au réel. Ensuite, parce qu'en choisissant de faire «avec» l'espace et pas seulement «sur » l'espace, elle se démarque des sorties sur le terrain ordinaire. Loin d'une sortie-conférence ou d'une sortie-questionnaire, la sortie sensible s'appuie sur ce qui se produit ici et maintenant, en mobilisant différents procédés donnant lieu à des sorties-jeu, des sorties connectées, des sorties-dérive, des balades sonores.

The experiential field trip is an original practice in schools for two reasons. First, by concentrating on "open air" geography (Chevalier, 2007), it contrasts with "workshop geography" during which documents, selected by the instructor and analysed within the classroom replace the real world. Second, by choosing to work "with" space and not just "on" space, it contrasts with more normalised field trips. Far from Lecture-Field trips or Questionnaire-Field trips, the experiential field trip is based on what happens in the here and now and uses various processes that give way to Play-field trips, Connected-field trips, Derivatedfield trips, soundwalks.

La salida de campo sensible es una práctica escolar doblemente original. En primer lugar porque favorece una geografía "al aire libre" (Chevalier, 2007) que se aleja de la clase magistral en la que la realidad es sustituída por documentos seleccionados por el profesor y analizados dentro del aula. En segundo lugar, porque se diferencia de las excursiones tradicioneales por su trabajo "en" 
el espacio y no "sobre" el espacio. Lejos de una salida expositiva o recopilatoria la salida de campo sensible se fundamenta en lo que está sucediendo en un territorio aquí y ahora, movilizando procedimientos para que se transite en ese entorno con juegos, buscando conexiones, realizando una movilidad a la deriva o recorridos sonoros.

\section{INDEX}

Thèmes : Réinventer les territoires par l'art - repenser l'art par les territoires

Mots-clés : sortie sensible, praticien chercheur, pratiques ordinaires, sortie-jeu, sortie connectée, sortie-dérive, balade sonore, sortie-conférence, sortie-questionnaire

Keywords : experiential field trips, practician-researcher, ordinary practices, Play field trips, connected field trips, derivated field trips, soundwalks, lecture field trips, questionnaire field trips

Palabras claves : salida sensible, investigador, prácticas habituales, juegos en el espacio, búsqueda de interrelaciones, paseos a la deriva, recorridos sonoros, clases en el campo, salidas para conocer 